\title{
Costruzione e Validazione del questionario ISCOS: uno studio preliminare sugli Indicatori di Sviluppo del COunselling Scolastico
}

\section{Giovanna Esposito $^{1}$ - Maria Francesca Freda ${ }^{2}$ Letizia Servillo ${ }^{3}$}

${ }^{1}$ Università degli Studi di Napoli «Federico II», Centro SInAPSi

${ }^{2}$ Università degli Studi di Napoli "Federico II", Dipartimento di Studi Umanistici

${ }^{3}$ Psicologa

doi: 10.7358/ecps-2013-008-espo

giovan.esposito@unina.it

fmfreda@unina.it

servilloletizia@libero.it

\section{CONSTRUCTION AND VALIDATION OF THE ISCOS QUESTIONNAIRE: A STUDY ON SCHOOL COUNSELLING DEVELOPMENT INDICATORS}

\section{Abstract}

The aim of the study is to construct and validate a new questionnaire called ISCOS (Development Indicators of School COunselling) designed to measure specific usage profiles of counselling at school. These profiles related to couselling service are called Provoke Profile, Distrust Profile, Fear Profile and Trust Profile, as identified in previous exploratory researches (Freda et al., 2009; Esposito et al., 2010). These profiles have been conceptualized as symbolic and cultural models (Carli \& Paniccia, 2003 and 2011) of the service representation. Another aim of the study is to investigate possible associations among these usage models and the influences of variables such as age and gender of the students. ISCOS was submitted to a number of 241 students (Female $=115 ;$ Male $=126$ ) aged among 13 and 20 years $(M=16.23 ; d s=1.81)$, attending a high school in Naples where a counselling service was already activated. The exploratory factor analysis showed the stability of two 
factors (Types of Investment and Retreat) that explain 37\% of the total variance. Finally, for both factors was found a significant difference concerning only the gender variable. The questionnaire showed good psychometric qualities. It presents itself as an innovative tool designed to analyze specific emotional dimensions underlying the usage models identified. However, further investigations are deemed necessary to further diversifying and expanding the sample with regard to the origin and the field of studies.

Keywords: Prevention, Questionnaire, School Counselling, Usage profiles, Validation.

\section{INTRODUZIONE}

Il counselling si sviluppa all'interno dell'istituzione scolastica come una risorsa, soprattutto preventiva, volta a rendere più consapevoli gli studenti dei propri disagi, sia di quelli prettamente connessi al mondo scolastico, sia di quelli relativi a particolari situazioni affettive e relazionali (Eyo, Joshua, \& Esuong, 2010; Chireshe, 2011). Come sottolinea Maggiolini (2006), «il counselling agisce soprattutto rafforzando la consapevolezza individuale sui problemi, che è una delle principali vie attraverso cui opera la prevenzione, accanto alla promozione di condizioni sociali, economiche e ambientali che favoriscono la salute e all'incoraggiamento del ricorso al sostegno psicosociale nei servizi». Uno degli obiettivi principali del counselling scolastico è, quindi, quello di favorire una cultura della prevenzione (Di Fabio, 2003), volta a promuovere all'interno dell'istituzione scolastica azioni concrete per migliorare la qualità della vita mentale e fisica dei suoi studenti (Lunenburg, 2010).

In linea con tale ottica preventiva, negli ultimi anni si è assistito all'attivazione di numerosi servizi di counselling scolastico in Europa e nel mondo e, parallelamente, si è avvertita la necessità su più fronti di valutarne l'efficacia in termini di miglioramento sia del benessere psicologico degli studenti sia della loro performance scolastica (Lonborg \& Bowen, 2004; Cooper et al., 2010). Ciò al fine anche di giustificare la consistente spesa pubblica che i governi hanno investito per l'attivazione di questi servizi e che, di fatto, non sempre, riescono a diventare luoghi preferenziali per le richieste di aiuto degli studenti. Si registrano, infatti, di frequente, bassi tassi di partecipazione al servizio, nonché, in caso di frequentazione, una scarsa efficacia dello stesso nel migliorare la salute psicofisica degli studenti o la loro performance scolastica (Vogel, Wade, \& Hackler, 2007). Una recente indagine metanalitica effettuata in America, ad esempio, ha evidenziato una diversa efficacia dei servizi di counselling sulla salute psicofisica degli studenti partecipanti e sul rischio di drop-out scolastico: efficacia variabile in base al genere, all'età dei 
partecipanti, ma anche alle competenze del counsellor (Whiston et al., 2011). Tali studi di valutazione di efficacia, inoltre, hanno utilizzato strumenti di misura diversi, alcuni validati e somministrati in differenti contesti nazionali (Fairchild \& Seeley, 1996; Cooper et al., 2010; McKenzie et al., 2010), altri messi a punto ad hoc per gli obiettivi del singolo studio (Eobochuku, 2008; Chireshe, 2010). In particolare, tra gli strumenti con migliori qualità psicometriche utilizzati in più ricerche si annoverano il CSE (Counselling Service Evaluation) (Fairchild \& Seeley, 1996), il YP-CORE (Young Persons Clinical Outcomes for Routine Evaluation) (Twigg et al., 2009) o l'ESQ (Experience of Service Questionnaire) (Bury NHS Trust, 2010). Altri studi di valutazione, invece, si sono avvalsi di strumenti di natura qualitativa, come interviste sull'esperienza di counselling effettuata (Prior, 2011) o metafore per la valutazione del counsellor (Ozabaci, 2011). Si tratta, in entrambi i casi, di strumenti di valutazione di esito del servizio volti a indagare, mediante domande chiuse o aperte, alcune dimensioni specifiche, come il benessere psico-emotivo degli studenti partecipanti, la motivazione ad utilizzare efficacemente il servizio, la competenza del counsellor ad accogliere e a sostenere gli utenti, i benefici ottenuti in termini di miglioramento della performance scolastica e, in generale, il livello di soddisfazione dell'esperienza effettuata.

In altre ricerche appartenenti a questo ampio filone di studi di efficacia del servizio, si è cercato, inoltre, di mettere in relazione gli esiti del counselling con le principali problematiche/richieste che hanno indotto gli studenti a parteciparvi (Boldero \& Barry, 1995; Busani, 2005; Madera, 2007; Bryan et al., 2009). In linea generale, questi studi rivelano che la maggior parte degli alunni chiede aiuto per problemi che riguardano la famiglia, le relazioni interpersonali, la salute, la droga e l'alcool, la depressione, il suicidio, la sessualità e l'educazione. Inoltre, è anche interessante sottolineare che le ragazze, che si rivolgono al servizio più spesso rispetto ai ragazzi, riportano maggiormente problematiche riguardanti la famiglia, le relazioni interpersonali e i problemi di salute, mentre i ragazzi tendono a riportare soprattutto problematiche riguardanti l'educazione (Dubow, Lovko, \& Kausch, 1990; Schonert-Reichl \& Muller, 1996).

Accanto a questo filone di studi di efficacia, condotti con studenti che hanno usufruito del servizio, è possibile individuare un altro filone di ricerche che invece si è concentrato sull'analisi delle attitudini degli studenti a partecipare al counselling e, più in generale, a ricercare un aiuto psicologico professionale nei counsellor operanti presso i vari contesti scolastici (Winograd \& Shick Tryon, 2009; Goh et al., 2007; Takamura et al., 2008; Wilson et al., 2005; Sayal, 2006; Ajzen, 2002; Raviv et al., 2000; Kuhl, Jarkon-Horlick, \& Morrissey, 1997). Anche in questo ambito si annoverano strumenti ampiamente utilizzati in diversi contesti che hanno mostrato buone qualità psico- 
metriche, come il BASH (Barriers to Adolescents Seeking Help) (Kuhl, JarkonHorlick, \& Morrissey, 1997), il GHSQ (General Help-Seeking Questionnaire) (Wilson et al., 2005), il ASPH (Attitudes toward Seeking Professional Psychological Help Scale) (Fisher \& Farina, 2005), o strumenti costruiti ad hoc per gli obiettivi del singolo studio, come il SSATGES (Secondary School Students' Attitude Towards Guidance and Counselling Service Questionnaire) (Eyo, Joshua, \& Esuong, 2010). Anche all'interno di questo filone si annoverano, inoltre, studi che si sono avvalsi di strumenti di rilevazione qualitativi, come interviste (Prior, 2011) o vignette cliniche (Raviv et al., 2000). Questi strumenti sono stati somministrati a studenti che avevano concretamente usufruito del servizio al fine di indagare le principali barriere psico-sociali alla richiesta di aiuto professionale, nonché il legame tra attitudini positive e negative verso il servizio e variabili come il genere, la provenienza geografica, l'autostima, lo stile attribuzionale, il precedente contatto con il servizio e la conoscenza pregressa sul counselling e sui servizi psicologici in generale (Winograd \& Shick Tryon, 2009). In tal senso, l'attitudine verso il servizio è stata messa in relazione, non tanto con le principali richieste rivolte al counselling, quanto con la percezione che gli utenti hanno dello stesso counselling scolastico che può contribuire ad influenzare attitudini positive e negative (West et al., 1991; Ciarrochi et al., 2003; Sheffield, Fiorenza, \& Sofronoff, 2004; Wilson et al., 2002; Rickwood, Deane, \& Wilson, 2007; Egbochuku, 2008; Ngale, 2009). Un risultato comune alle diverse ricerche è la preoccupazione da parte degli adolescenti per la riservatezza dei motivi che li hanno indotti a rivolgersi allo sportello, la paura di non essere compresi, di continuare ad essere dipendenti da un adulto, di essere etichettati dagli altri e di dover raccontare problemi personali ad una persona estranea che non può comprenderli come farebbe una persona più vicina. Ciò che d'interessante emerge in queste ricerche è che una minore frequentazione degli sportelli è da ricollegare ad una rappresentazione del servizio come luogo in cui si recano solo e prevalentemente i ragazzi che vivono seri stati di disagio (Gattini, 2005), in altre parole, come luogo della malattia mentale che rischia di etichettare lo studente che vi partecipa (Rickwood, Deane, \& Wilson, 2007; Prior, 2011).

Gli studi afferenti ad entrambi i filoni descritti, pur avvalendosi di strumenti di misura spesso ampiamente validati e consolidati nella letteratura internazionale e nazionale, ricollegano tuttavia rispettivamente l'efficacia del servizio o la propensione a prendervi parte a caratteristiche prevalentemente individuali (come il genere, l'età, le problematiche dello studente, la sua motivazione, ecc.) o ambientali/situazionali (come la funzionalità del servizio, le competenze del counsellor, ecc.). Essi assumono che le variabili esaminate incidano sull'efficacia del servizio o sull'attitudine a parteciparvi in modo invariante, indipendentemente cioè dal contesto socio-culturale nel quale l'im- 
patto di queste variabili è misurato. Tuttavia, noi assumiamo che dimensioni individuali e situazionali giochino un ruolo diverso al variare dei significati che prendono forma nella relazione tra studenti e servizio all'interno di contesti culturali specifici. Gli studenti partecipano alla costruzione e usano tali significati per mediare le proprie motivazioni e modalità di partecipazione al servizio di counselling, per studiare il fenomeno abbiamo bisogno di modelli in grado di cogliere tale relazione ed i processi di significazione che la attraversano (Valsiner \& Rosa, 2007; Salvatore \& Valsiner, 2010). Tali processi di significazione non sono costruzioni esclusivamente individuali ma sono frutto di attività di negoziazione intersoggettiva all'interno di un contesto culturale e simbolico (Candela, 2005; Iannaccone et al., 2012; Venuleo et al., 2008; Linell, 2009; Salvatore, 2012). Assumiamo, quindi, che gli studenti che afferiscono al servizio di counselling lo interpretino sulla base ed entro i vincoli dei set di significati condivisi e negoziati nel loro contesto.

Tali premesse ci consentono di introdurre un terzo filone di studi volto ad esplorare le dinamiche collusive o affettive (Carli, Paniccia, \& Salvatore, 2004) organizzanti la relazione dello studente con il servizio di counselling. Tali dinamiche inscrivono il counselling in un contesto simbolico-emozionale, entro il quale il servizio acquista uno specifico significato condiviso da sottogruppi di studenti. Il filone che proponiamo è interessato a rilevare, quindi, processi di rappresentazione culturale del servizio, attraverso cui i diversi sottogruppi di soggetti intenzionano emozionalmente il contesto a cui partecipano (Carli \& Paniccia, 2003; Salvatore \& Scotto di Carlo, 2005). La rappresentazione culturale trae origine dalla concettualizzazione di Culture Locali, vale a dire sistemi collusivi contestualizzati e storicizzati, caratteristici di specifici contesti (Carli \& Paniccia, 2003). La Cultura Locale indica il processo di simbolizzazione affettiva condivisa dai diversi partecipanti ad un contesto e, quindi, una rappresentazione dei loro scopi e, più in generale, del senso delle loro azioni e del loro rapporto. Tuttavia, all'interno del medesimo contesto, si possono istituire Modelli Culturali differenti di rappresentazione della realtà che caratterizzano specifici sottogruppi di partecipanti al contesto (Salvatore \& Scotto di Carlo, 2005). Lo studente, pertanto, ha un certo grado di autonomia nell'interpretazione del Modello Culturale cui prende parte. Questo significa che un Modello Culturale può essere studiato a livello individuale, esplorando il tipo di significati culturali assunti da insiemi di studenti, significati che orientano il modo con cui gli studenti percepiscono, vivono e si rapportano al servizio di counselling, regolando, ad esempio, l'investimento espresso, la loro attitudine o l'utilizzo che ne fanno in termini di miglioramento del proprio benessere psicofisico o del rendimento scolastico.

Entro questo filone si registrano studi volti ad analizzare in generale la modalità di rappresentazione culturale dell'immagine dello psicologo (Carli 
\& Salvatore, 2001; Carli, Paniccia, \& Salvatore, 2004) e della sua professione (Giovagnoli, Dolcetti, \& Paniccia, 2008; Carli \& Paniccia, 2011), delle sue ricadute nei diversi contesti professionali in cui opera (Carli et al., 2009; Dolcetti et al., 2006; Giovagnoli, Giuliano, \& Paniccia, 2009). Con riferimento specifico al counselling scolastico si annoverano gli studi, da alcuni di noi condotti, che si sono tuttavia avvalsi di strumenti di rilevazione qualitativi, come le vignette (Freda, Esposito, \& La Montagna, 2009; Esposito, Freda, \& Franzese, 2010). A differenza dei primi due filoni descritti in precedenza, mancano in quest'ambito questionari quantitativi di rilevazione dei Modelli Culturali di rappresentazione del counselling che consentano di analizzare tali dinamiche collusive/affettive su larga scala in ragione dei contesti di rilevazione. Riteniamo, infatti, che sia necessario rilevare tali rappresentazioni culturali che vengano a connotare il rapporto tra clienti e servizio di counselling al fine di promuovere uno spazio funzionale al miglioramento della prestazione offerta e della qualità della relazione stessa e orientare gli studenti ad usufruire del servizio qualora ne riconoscano l'opportunità ed il bisogno. Nostro interesse, in particolare, è esplorare tali rappresentazioni, non tanto con sottogruppi di studenti che hanno concretamente usufruito del servizio, ma con tutta quella popolazione studentesca che conosce il counselling in quanto attivo presso la propria scuola di appartenenza, ma ancora non vi si è rivolto. Riteniamo che sia importante esplorare anche un'ipotetica fruibilità del counselling, modelli di uso del servizio in potenziali clienti, ciò al fine di agevolare coloro che investono su questo importante spazio d'ascolto nel progettare interventi che possano concretamente analizzare la domanda di quella particolare scuola e dei suoi potenziali clienti (Esposito, Zaccaro, \& Freda, 2009).

\section{Obiettivi}

In linea con il terzo filone di studi appena descritto, la presente ricerca si propone l'obiettivo di costruire e validare un nuovo questionario finalizzato ad eplorare Modelli Culturali di uso del servizio di counselling. Si assume, infatti, che tali modelli, già emersi in precedenti ricerche di tipo qualitativo (Freda, Esposito, \& La Montagna, 2009; Esposito, Freda, \& Franzese, 2010), possano favorire o ostacolare la propensione dello studente a rivolgersi al servizio stesso, nonché l'efficacia del counselling in termini di miglioramento della salute psico-fisica dello studente e della sua performance scolastica.

Lo studio che presentiamo è un iniziale pretest di validazione dello strumento che ha lo scopo di fornire eventuali indicazioni operative per una successiva ridefinizione degli item e della messa a punto del questionario stesso in ulteriori contesti scolastici. 
Ulteriore obiettivo è indagare eventuali associazioni tra tali Modelli Culturali di uso e alcune variabili socio-anagrafiche come l'età e il genere degli studenti.

\section{LA FASE PRELIMINARE QUALITATIVA}

La presente ricerca di validazione si basa sui risultati ottenuti da due studi esplorativi precedenti (Freda, Esposito, \& La Montagna, 2009; Esposito, Freda, \& Franzese, 2010) il cui obiettivo era individuare profili di utilizzo del counselling individuale a scuola. Tali profili, intesi come modelli simbolici e culturali (Carli \& Paniccia, 2003 e 2011) di rappresentazione del servizio di counselling, sono stati indagati mediante un kit di vignette con la consegna di scrivere nelle nuvolette ciò che il protagonista stesse dicendo o pensando. Tali vignette, denominate L'ENTRATA, L'INCONTRO e L'USCITA, riproducevano il processo di relazione tra un ipotetico studente e il servizio di counselling, a partire dalla sua fase istituente fino a quella conclusiva. Nello specifico, la vignetta L'ENTRATA si proponeva di sollecitare quelle rappresentazioni associate alla disponibilità del servizio di counselling. La vignetta LINCONTRO voleva essere uno stimolo alle rappresentazioni inerenti il processo di counselling. Infine, L'USCITA si proponeva di sollecitare l'espressione delle reazioni del protagonista che aveva appena concluso l'incontro e usciva dalla stanza di counselling ${ }^{1}$.

Entrambe le ricerche esplorative sono state condotte in scuole in cui è attivo da diversi anni un servizio di counselling individuale rivolto agli studenti e coordinato da alcuni psicologi operanti presso l'ASL del territorio. Tutte le ricerche, tra cui anche l'attuale, sono state condotte in contesti scolastici sempre diversi.

La metodologia d'analisi dei dati, utilizzata in entrambe le ricerche, è stata di natura quali-quantitativa (Bolasco, 1999; Cicognani, 2003). Nello specifico, mediante l'ausilio del software SPHINKS (versione Lexica), è stata effettuata innanzitutto un'Analisi del Contenuto di III tipo (Losito, 1993) sulle narrazioni fornite dai soggetti alle vignette; ciò al fine di rilevare possibili categorie di uso del servizio da parte degli studenti partecipanti alla ricerca.

Successivamente è stata effettuata un'Analisi delle Corrispondenze Multiple (ACM) che ha utilizzato le categorie di uso come variabili attive e dalla quale sono emersi due fattori ${ }^{2}$.

1 Si rimanda al questionario in Appendice per prendere visione delle vignette utilizzate.

2 Il Primo Fattore, quello orizzontale, esprime l'atteggiamento nei confronti del servizio di counselling individuale e che può essere pensato come un continuum che va da un'attrazione 
Infine, è stata effettuata un'interpretazione geometrico-strutturale (Bolasco, 1999) che ha consentito di individuare quattro modelli di utilizzo del counselling individuale. Nello specifico, il primo modello, il Modello dell' $A f-$ fidarsi, denota un tipo di studente che ipotizza di rivolgersi al counsellor, inteso come un esperto disponibile, per essere ascoltato e contenuto. Il Modello del Temere identifica, invece, un tipo di studente che, pur riconoscendo il suo bisogno di aiuto, si sente paralizzato dalle ansie e dalle angosce relative all'incontro con il counsellor, sentito come un esperto giudicante. Il Modello del Diffidare connota un tipo di studente che ha perso la fiducia nell'altro ed evita di cimentarsi in nuove relazioni per timore di spiacevoli sorprese. Ed, infine, il Modello del Provocare riguarda un tipo di studente che, pur riconoscendo al counsellor una competenza ed una disponibilità all'aiuto, esprime nei suoi confronti un atteggiamento di rifiuto, di critica e di attacco.

\section{Metodo}

\subsection{Soggetti}

La presente ricerca è stata svolta in un istituto di Scuola Media di secondo grado ${ }^{3}$ ubicato in un quartiere del centro storico della città di Napoli. Nell'istituto è attivo da diversi anni un servizio di counselling individuale ad orientamento psicodinamico, rivolto agli studenti e coordinato da alcuni psicologi operanti presso l'U.O.P.C.E.E. ${ }^{4}$ del territorio. Il gruppo di soggetti partecipanti alla ricerca è composto da 241 studenti $(F=115 ; \mathrm{M}=126)$ con un'età compresa tra i 13 e i $20 \mathrm{anni}^{5}(\mathrm{M}=16.23$; $\mathrm{ds}=1.81)$ e frequentanti le classi prime $(32.8 \%)$, terze $(32.4 \%)$ e quinte $(34.9 \%)$ dell'indirizzo scientifico ${ }^{6}$.

verso il servizio (estremo sinistro) alla sua repulsione (estremo destro). Il Secondo Fattore, quello verticale, è espressione della relazione che l'ipotetico fruitore instaura con l'adulto, con un counsellor percepito sia come un esperto disponibile (nell'estremità superiore dell'asse), sia come un esperto giudicante (nell'estremità inferiore).

3 La scuola è il Liceo Statale «Eleonora Pimentel Fonseca»; al Dirigente Scolastico, ai docenti e agli studenti che hanno collaborato alla raccolta dati va il nostro più sentito ringraziamento.

4 Unità Operativa di Psicologia Clinica e dell'Età Evolutiva, ASL NA 1, Distretto 51. Ai responsabili del servizio che hanno supportato la ricerca va il nostro più sentito ringraziamento per la disponibilità mostrata durante tutte le fasi del processo di ricerca.

5 I soggetti sono stati suddivisi rispetto alle diverse età in tre gruppi: soggetti con età compresa dai 12 ai 14 anni (39\%), dai 15 ai 17 anni (29\%) e dai 18 ai 20 anni (38\%). Tali fasce d'età scandiscono specifiche fasi del periodo adolescenziale: pre-adolescenza, adolescenza e tarda adolescenza (Blos, 1962).

6 Queste classi sono state scelte in quanto segnano tappe importanti nella vita dello studente. La prima classe segna, infatti, il passaggio dalle scuole medie a quelle superiori. La terza 
Nello specifico l'età media delle partecipanti di sesso femminile è di 16.3 con una deviazione standard di 2.2. L'età media dei partecipanti di sesso maschile è di 16 con una deviazione standard di 1.8. Al test del $\chi 2$ non sono risultate differenze significative per quanto riguarda il genere $(\chi 2=.50 ; \mathrm{gdl}=1 ; p=.48)$, la classe $\left(\chi^{2}=.26 ; \mathrm{gdl}=2 ; p=.88\right)$ ed, infine, l'età $(\chi 2=3.62 ; \mathrm{gdl}=2 ; p=.16)$.

Inoltre, la maggioranza dei genitori dei soggetti è in possesso di un diploma di scuola media superiore (51\% delle madri e $39 \%$ dei padri) ${ }^{7}$ e svolge un lavoro da impiegato ( $45 \%$ delle madri e $66 \%$ dei padri) ${ }^{8}$.

\subsection{Strumenti}

È stato costruito e somministrato, secondo la metodologia ISO (Indicatori di Sviluppo Organizzativo) (Carli, Paniccia, \& Salvatore, 2004; Salvatore \& Scotto di Carlo, 2005; Carli \& Paniccia, 2011), un questionario indicato con la sigla ISCOS (Indicatori di Sviluppo del COunselling Scolastico).

Tale metodologia è stata elaborata da SPS - Studio di Psicosociologia - e utilizzata in diverse indagini culturali, sia entro i sistemi organizzativi che nei contesti sociali (Carli et al., 1997; Carli \& Salvatore, 2001; Carli \& Paniccia, 2002; Cavalieri, 2003; Carli, Paniccia, \& Salvatore, 2004; Carli \& Paniccia, 2011) al fine di individuare la Cultura Locale e i Modelli Culturali presenti in uno specifico contesto. In generale, lo scopo dell'analisi ISO è la clusterizzazione del campione in funzione di specifici profili di risposta; infatti, l'analisi dei dati è volta a differenziare il campione in gruppi di soggetti tra loro simili nel rispondere alle domande proposte dal questionario che fanno riferimento a specifici Modelli Culturali. È importante rilevare, inoltre, che nella costruzione del questionario ISO, la definizione delle domande e delle alternative di risposta non si basa sul criterio della rappresentatività ed esaustività di tutti i possibili aspetti dell'oggetto di studio, bensì, come sottolineano Salvatore e Scotto di Carlo (2005), fa riferimento a «opzioni semantiche indicative di specifiche modalità di connotazione simbolica dell'oggetto, rilevanti sul pia-

classe è stata pensata, invece, come un momento di passaggio tra la fanciullezza e l'imminente maggiore età. La quinta classe, infine, segna un nuovo momento di passaggio, di entrata ormai imminente nella gioventù.

7 Livello di istruzione delle madri: licenza elementare (4\%), licenza media (20\%), diploma di laurea $(22 \%)$ e specializzazione post-lauream (3\%). Livello di istruzione dei padri: licenza elementare (4\%), licenza media $(25 \%)$, diploma di laurea $(30 \%)$ e specializzazione postlauream $(2 \%)$.

8 Altre professioni svolte dalle madri: casalinga (37\%), operaio (2\%), imprenditore $(4 \%)$, commerciante $(6 \%)$, disoccupato $(6 \%)$. Altre professioni svolte dai padri: operaio (11\%), imprenditore (8\%), commerciante (11\%), disoccupato (3\%) e pensionato (1\%). 
no culturale, che permettono di giungere a definire specifici profili di risposta». Ne consegue, dunque, che se un determinato gruppo di persone si caratterizza per un certo tipo di risposte al questionario o profilo di risposta ciò dipende da un certo modo di pensare, da una certa mentalità, che dà ragione di tale co-occorrenza (Salvatore \& Scotto di Carlo, 2005). Non è necessaria una conoscenza reciproca tra le persone che prendono parte alla ricerca per evidenziarne l'appartenenza ad uno stesso Modello Culturale; questi ultimi, infatti, si manifestano grazie al fatto che le persone interpellate condividono il medesimo contesto collusivo dato dall'oggetto della ricerca stessa.

\subsubsection{Il questionario ISCOS}

Il questionario ISCOS è stato costruito per analizzare, non tanto il funzionamento del servizio di counselling, quanto la cultura locale del servizio stesso e gli specifici Modelli Culturali di utilizzo condivisi da sottogruppi di studenti. Nello specifico, il questionario è costituito da ventiquattro item tesi a misurare i quattro modelli di utilizzo del counselling individuale a scuola, rilevati nelle ricerche precedenti (Freda, Esposito, \& La Montagna, 2009; Esposito, Freda, \& Franzese, 2010) e definiti del Provocare, Diffidare, Temere e Affidarsi al servizio.

In particolar modo, per la costruzione degli item, sono state selezionate, con l'aiuto di due ricercatori, di cui uno cieco, quelle narrazioni riportate dagli studenti nelle vignette della seconda ricerca esplorativa (Esposito, Freda, $\&$ Franzese, 2010), che avevano contribuito in maniera significativa all'individuazione delle categorie più rappresentative di ciascun modello. Nello specifico sono state scelte sei narrazioni per ogni modello. Tali modelli sono stati così trasformati in vere e proprie scale di misurazione di specifiche dinamiche simbolico-culturali sottese alla relazione tra studente e servizio di counselling dalla sua fase istituente fino a quella conclusiva (Tabella 1).

Inoltre, per favorire processi proiettivi e identificativi, gli item sono stati accompagnati dalle tre vignette usate nelle ricerche precedenti (LENTRATA, L'INCONTRO e L'USCITA: due item di ogni scala per ciascuna vignetta) e da una specifica consegna, nella quale si descrive l'azione del/la protagonista ${ }^{9}$ e si chiede ai partecipanti di mettersi nei suoi panni e di esprimere, per ogni affermazione, il proprio grado di accordo o disaccordo su una scala Likert a cinque punti (da 1 «Per niente d'accordo» a 5 «Moltissimo d'accordo») ${ }^{10}$.

9 Il questionario è stato realizzato nella doppia versione maschile e femminile al fine di favorire i processi identificativi e proiettivi.

10 Si rimanda al questionario in Appendice. 
Tabella 1. - Gli item delle scale.

\begin{tabular}{|c|c|c|c|c|}
\hline $\begin{array}{c}\text { SCAle } \\
\text { VIGNETTE }\end{array}$ & AFFIDARSI & TEMERE & PROVOCARE & DIFFIDARE \\
\hline \multirow[t]{2}{*}{ L'ENTRATA } & $\begin{array}{l}\text { Item 2: } \\
\text { Ecco, è il momento } \\
\text { giusto per parlare } \\
\text { dei miei problemi } \\
\text { esfogarmi } \\
\text { senza essere } \\
\text { giudicatalo. }\end{array}$ & $\begin{array}{l}\text { Item 1: } \\
\text { Che imbarazzo!! } \\
\text { Chissà cosa penserà } \\
\text { di me! }\end{array}$ & $\begin{array}{l}\text { Item 4: } \\
\text { Entro, ma tanto non } \\
\text { ho nessun problema! }\end{array}$ & $\begin{array}{l}\text { Item 3: } \\
\text { Voglio proprio vedere } \\
\text { cosa mi dice! }\end{array}$ \\
\hline & $\begin{array}{l}\text { Item 7: } \\
\text { Finalmente qualcuno } \\
\text { che mi può aiutare } \\
\text { a capire meglio i miei } \\
\text { pensieri. }\end{array}$ & $\begin{array}{l}\text { Item 6: } \\
\text { Che vergogna! } \\
\text { Speriamo che nessuno } \\
\text { mi veda entrare. }\end{array}$ & $\begin{array}{l}\text { Item 8: } \\
\text { Entriamo va, cosi } \\
\text { perdo l'ora di lezione! }\end{array}$ & $\begin{array}{l}\text { Item 5: } \\
\text { Mah! Chissà se } \\
\text { riusciranno } \\
\text { ad aiutarmi. }\end{array}$ \\
\hline \multirow{2}{*}{ L'INCONTRO } & $\begin{array}{l}\text { Item 11: } \\
\text { Buongiorno! Ho } \\
\text { un problema, non so } \\
\text { cosa fare e vorrei } \\
\text { un consiglio. }\end{array}$ & $\begin{array}{l}\text { Item 12: } \\
\text { Mi vergogno un po' } \\
\text { di esprimere le mie } \\
\text { emozioni e raccontare } \\
\text { di me: speriamo } \\
\text { di non fare brutte } \\
\text { figure. }\end{array}$ & $\begin{array}{l}\text { Item 9: } \\
\grave{E} \text { inutile parlare, } \\
\text { tanto mi dicono tutti } \\
\text { le stesse cose. }\end{array}$ & $\begin{array}{l}\text { Item 10: } \\
\text { Chissà se posso } \\
\text { fidarmi! }\end{array}$ \\
\hline & $\begin{array}{l}\text { Item 16: } \\
\text { Mi fa piacere parlare } \\
\text { con una persona } \\
\text { che ha più esperienza } \\
\text { di me. }\end{array}$ & $\begin{array}{l}\text { Item 13: } \\
\text { Che ansia, speriamo } \\
\text { che mi faccia qualche } \\
\text { domanda perché } \\
\text { io non so proprio } \\
\text { da dove incominciare. }\end{array}$ & $\begin{array}{l}\text { Item 14: } \\
\text { A lei non dico } \\
\text { la verità, perché } \\
\text { dei miei problemi ne } \\
\text { parlo con la mia } \\
\text { famiglia o con i miei } \\
\text { amici, mi capiscono } \\
\text { sicuramente di più. }\end{array}$ & $\begin{array}{l}\text { Item 15: } \\
\text { Non so se posso } \\
\text { aprirmi, in fondo } \\
\text { è una persona che } \\
\text { non conosco! }\end{array}$ \\
\hline \multirow{2}{*}{ L'USCITA } & $\begin{array}{l}\text { Item 18: } \\
\text { Mi sento più } \\
\text { rilassata e con le idee } \\
\text { più chiare. }\end{array}$ & $\begin{array}{l}\text { Item 20: } \\
\text { Non ce l'ho fatta } \\
\text { proprio a parlare } \\
\text { anche se volevo } \\
\text { sfogarmi. Avevo } \\
\text { troppa vergogna. }\end{array}$ & $\begin{array}{l}\text { Item 17: } \\
\text { Lo sapevo non è } \\
\text { servito a niente. } \\
\text { Sto peggio di prima. }\end{array}$ & $\begin{array}{l}\text { Item 19: } \\
\text { Non so se la prossima } \\
\text { volta ritornerò. }\end{array}$ \\
\hline & $\begin{array}{l}\text { Item 23: } \\
\text { Mi sento sollevata ora } \\
\text { che ho parlato } \\
\text { dei miei problemi } \\
\text { invece di tenermeli } \\
\text { dentro. }\end{array}$ & $\begin{array}{l}\text { Item 24: } \\
\text { Che imbarazzo, } \\
\text { non sapevo proprio } \\
\text { che dire! }\end{array}$ & $\begin{array}{l}\text { Item 22: } \\
\text { Ab! Meno male che } \\
\text { è finita! Mi stavo } \\
\text { proprio annoiando! }\end{array}$ & $\begin{array}{l}\text { Item 21: } \\
\text { Mi sento scettico } \\
\text { chissà per quale } \\
\text { motivo fanno } \\
\text { queste sedute. }\end{array}$ \\
\hline
\end{tabular}




\subsection{Procedura}

È stata richiesta l'autorizzazione alla somministrazione del questionario e il consenso informato da parte degli studenti. La somministrazione ha avuto luogo nell'a.s. 2009/2010 nei mesi di marzo/aprile durante le ore di lezione e ha richiesto circa 30 minuti per classe. Non sono stati richiesti elementi atti ad identificare i singoli soggetti, nel rispetto delle norme vigenti sulla privacy (L. 196/2003).

\section{ANALISI DEI DATI}

I dati concernenti le quattro scale per la misurazione dei modelli di utilizzo del servizio di counselling a scuola sono stati analizzati con l'ausilio del software SPSS 11 al fine di valutare: l'affidabilità e la struttura fattoriale delle singole scale in questione, la presenza di possibili correlazioni e, infine, le differenze di genere ed età. Per valutare l'affidabilità delle singole scale è stata effettuata una Reliability Analysis condotta attraverso il calcolo dell'alfa di Cronbach e della correlazione item-scala totale. Per la valutazione della struttura fattoriale è stata eseguita un'Analisi Fattoriale con il metodo di estrazione dei fattori principali (AFP) ${ }^{11}$ e rotazione OBLIMIN. Successivamente è stata eseguita un'analisi fattoriale esplorativa sempre con il metodo di estrazione dei fattori principali (AFP), nella quale sono stati inseriti tutti gli item delle singole scale. Al fine di analizzare differenze ascrivibili al genere e all'età è stata condotta un'Analisi della Varianza Multivariata (MANOVA) considerando come variabili indipendenti il genere e l'età dei soggetti e come variabili dipendenti i punteggi ottenuti nei fattori emersi dall'analisi fattoriale esplorativa.

\section{Risultati}

\subsection{Analisi dell'affidabilità}

La scala dell' Affidarsi presenta un alpha di Cronbach con valore pari a $.82 \mathrm{e}$ tutti gli item mostrano una correlazione item-scala superiore a .30 (Tabella 2). La soluzione fattoriale ottenuta spiega il $53 \%$ della varianza totale.

11 L'Analisi dei Fattori Principali (AFP) o Principal Axis Factoring (PAF) è una variante del modello in fattori comuni molto utilizzata, essa applica la «strategia» dell'analisi delle componenti principali, che presenta stime di comunalità $\left(\mathrm{R}_{2}\right)$ (Ercolani, Areni, \& Manetti, 1998). 
Tabella 2. - Correlazione item-scala della scala dell'Affidarsi.

\begin{tabular}{rcc}
\hline Item & $\begin{array}{c}\text { Correlazione } \\
\text { ITEM-SCALA TOTALE }\end{array}$ & $\begin{array}{c}\text { Alpha di Cronbach } \\
\text { SE L'ITEM Fosse ELIMiNATO }\end{array}$ \\
\hline $\mathbf{2}$ & $\mathbf{5 6}$ &, 79 \\
7 & $\mathbf{6 1}$ &, 78 \\
$\mathbf{1 1}$ & $\mathbf{6 7}$ &, 79 \\
$\mathbf{1 6}$ & $\mathbf{5 3}$ &, 79 \\
$\mathbf{1 8}$ & $\mathbf{5 6}$ &, 79 \\
$\mathbf{2 3}$ & $\mathbf{6 7}$ & 77 \\
\hline
\end{tabular}

La scala del Temere presenta un buon alpha di Cronbach pari a .79 e tutti gli item mostrano una correlazione item-scala superiore a .30 (Tabella 3). Dall'Analisi dei Fattori Principali è emersa una soluzione ad un solo fattore che spiega il $49,5 \%$ della varianza totale.

Tabella 3. - Correlazione item-scala della scala del Temere.

\begin{tabular}{|c|c|c|}
\hline ITEM & $\begin{array}{c}\text { CORRELAZIONE } \\
\text { ITEM-SCALA TOTALE }\end{array}$ & $\begin{array}{c}\text { Alpha di CRONBACH } \\
\text { SE L'ITEM FOSSE ELIMINATO }\end{array}$ \\
\hline 1 & ,58 & ,75 \\
\hline 6 & ,45 & ,79 \\
\hline 12 & ,63 & ,74 \\
\hline 13 & ,48 & ,77 \\
\hline 20 &, 52 & ,77 \\
\hline 24 & ,62 & 74 \\
\hline
\end{tabular}

La scala del Provocare presenta un valore dell'alpha di Cronbach sufficiente, convenzionalmente accettabile, pari a .65 , però non tutti gli item mostrano una correlazione item-scala superiore a .30. Nello specifico, gli item in questione sono l'item 4 (Entro, ma tanto non ho nessun problemal) e l'item 14 (A lei non dico la verità, perché dei miei problemi ne parlo con la mia famiglia o con $i$ miei amici, mi capiscono sicuramente di più). Si è proceduto, di conseguenza, ad eliminare per primo l'item 4 con correlazione item-scala più bassa (.26) rispetto all'item 14 (.28). La correlazione item-scala dell'item 14 è così aumentata, arrivando al di sopra del valore soglia. Tale delezione non ha comportato alcun cambiamento per quanto riguardo il valore dell'alpha di Cronbach che è risultato invariato (.65). La scala del provocare è, dunque, costituita definitivamente da 5 item (Tabella 4). La soluzione fattoriale ottenuta spiega il $43 \%$ della varianza totale. 
Tabella 4. - Correlazione item-scala della scala del Provocare.

\begin{tabular}{|c|c|c|}
\hline ITEM & $\begin{array}{c}\text { CORRELAZIONE } \\
\text { ITEM-SCALA TOTALE }\end{array}$ & $\begin{array}{c}\text { Alpha di CRONBACH } \\
\text { SE L'ITEM FOSSE ELIMINATO }\end{array}$ \\
\hline 8 & ,38 & ,62 \\
\hline 9 & ,43 &, 59 \\
\hline 14 & ,30 & ,64 \\
\hline 17 & ,44 &, 59 \\
\hline 22 &, 51 &, 54 \\
\hline
\end{tabular}

Infine, la scala del Diffidare presenta un valore di Cronbach inadeguato pari a .45 e solo due item (item 10 e 19) presentano una correlazione item-scala pari o superiore a .30 (Tabella 5). Si è, dunque, proceduto ad eliminare item per item, ma il valore dell'alpha è sempre risultato eccessivamente basso, per questo motivo la scala è stata del tutto eliminata dal questionario.

Tabella 5. - Correlazione item-scala della scala del Diffidare.

\begin{tabular}{|c|c|c|}
\hline ITEM & $\begin{array}{l}\text { CORRELAZIONE } \\
\text { ITEM-SCALA TOTALE }\end{array}$ & $\begin{array}{c}\text { Alpha di Cronbach } \\
\text { SE L'ITEM FOSSE ELIMINATO }\end{array}$ \\
\hline 3 & ,09 & ,49 \\
\hline 5 & ,11 & ,47 \\
\hline 10 & ,30 &, 36 \\
\hline 15 & ,28 & ,37 \\
\hline 19 & ,31 & ,37 \\
\hline 21 & ,27 & ,38 \\
\hline
\end{tabular}

\subsection{Analisi fattoriale esplorativa}

L'analisi fattoriale esplorativa, in cui sono stati inseriti tutti gli item delle singole scale, ha evidenziato la presenza di quattro componenti, ma sulla base dello scree-test (Figura 1) e prendendo in considerazione quelle componenti con autovalore superiore all'unità, si è deciso di estrarre due fattori che spiegano il 37\% della varianza totale. Dopo la rotazione OBLIMIN, la struttura fattoriale è apparsa di semplice interpretazione. I fattori sono stati interpretati considerando, come livello minimo, un valore di saturazione fattoriale superiore a .30 (Tabella 6). 


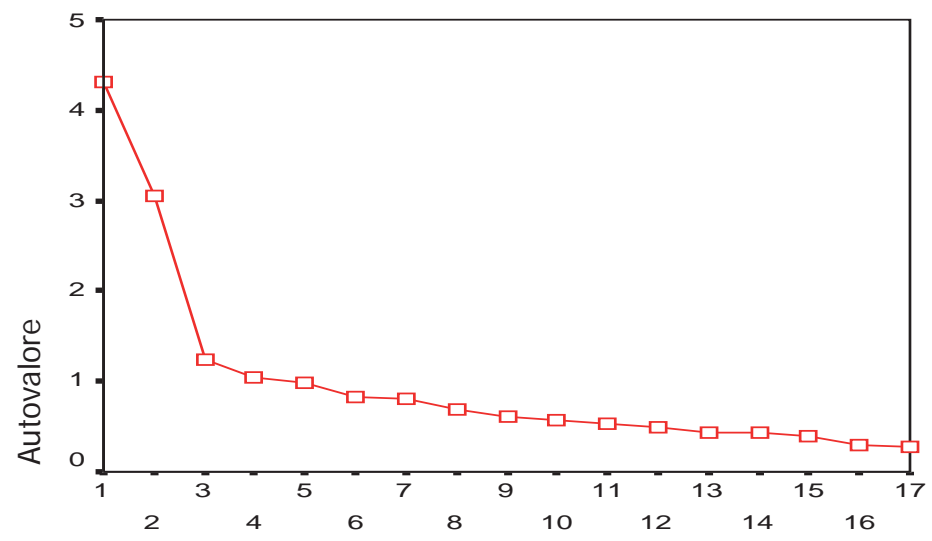

Fattore

Figura 1. - Scree-plot: grafico decrescente autovalori.

Tabella 6. - Rotazione OBLIMIN: saturazione dei fattori.

\begin{tabular}{|c|c|c|}
\hline ITEM & FATTORE 1 & FATTORE 2 \\
\hline 2 & ,620 & ,025 \\
\hline 7 & ,692 & ,052 \\
\hline 8 &,- 380 &,- 014 \\
\hline 9 &,- 499 & ,251 \\
\hline 11 & ,562 &,- 034 \\
\hline 14 &,- 359 & , 156 \\
\hline 16 & ,606 &,- 203 \\
\hline 17 &,- 484 & ,238 \\
\hline 18 & ,634 &,- 102 \\
\hline 22 &,- 598 & ,019 \\
\hline 23 & ,710 & ,082 \\
\hline 1 &,- 110 & ,665 \\
\hline 6 &,- 096 & ,493 \\
\hline 12 & ,073 & ,725 \\
\hline 13 & ,011 & ,538 \\
\hline 20 &,- 171 & ,613 \\
\hline 24 &,- 137 & ,715 \\
\hline
\end{tabular}


Il Primo Fattore spiega il 22\% della varianza totale e presenta item con saturazioni sia positive che negative. Nello specifico, gli item che contribuiscono positivamente al significato del fattore sono quelli che costituiscono la scala dell'Affidarsi, mentre quelli che contribuiscono negativamente sono quelli che appartengono alla scala del Provocare. Dopo, dunque, aver invertito il valore dei cinque item con saturazione fattoriale negativa, si è calcolato l'alpha di Cronbach, il cui valore è risultato pari a .82 .

Abbiamo denominato questo Primo Fattore Tipo di Investimento, poiché è costituito sia da item che esprimono il bisogno di chiedere aiuto ad una persona esperta, sia da item che esprimono un rifiuto di tale aiuto, che si palesa in un atteggiamento di critica e di disinteresse. Si riscontra, infatti, sempre un andare verso «l'altro» (il servizio e il counsellor) con un movimento attivo di attrazione (investimento di tipo positivo) o di repulsione (investimento di tipo negativo).

Il Secondo Fattore spiega il 15\% della varianza totale ed è del tutto sovrapponibile alla scala del Temere $(\alpha=.79)$. Abbiamo denominato questo Secondo Fattore Ritiro, in quanto caratterizzato da item che esprimono emozioni, quali l'ansia e la paura, connesse al giudizio e alla valutazione da parte dell'altro, che portano lo studente ad assumere un atteggiamento più passivo nei confronti del servizio. Infatti, gli item racchiusi in questo fattore, sebbene mostrino un voler andare verso "l'altro" in ragione di un riconoscimento del proprio bisogno, segnalano la presenza di ansie e angosce che si attivano nella relazione con il servizio e il counsellor.

\subsection{MANOVA: differenze di genere e di età}

Al fine di esplorare differenze ascrivibili al genere e all'età è stata condotta una MANOVA $2 \times 2$ considerando come variabili indipendenti il genere e l'età e come variabili dipendenti i punteggi ottenuti nei due fattori emersi dall'analisi fattoriale (Tipo di Investimento e Ritiro). Una volta verificato che le matrici di varianza/covarianza dei gruppi risultassero omogenee (Box's $M=$ $45.9 ; F=1.04 ; p=.41)$, il test multivariato ha mostrato una correlazione significativa solo per quanto riguarda la variabile genere in tutte e due i fattori (Tabella 7).

Nello specifico, per quanto riguarda il fattore Tipo di Investimento, le femmine riportano un livello significativamente superiore a quello dei maschi.

Anche per quanto riguarda il secondo fattore Ritiro, osservando le medie relative alla variabile genere, possiamo affermare che le ragazze esplicitano maggiormente emozioni di ansia, imbarazzo, timore di essere giudicate dagli altri rispetto ai ragazzi. 


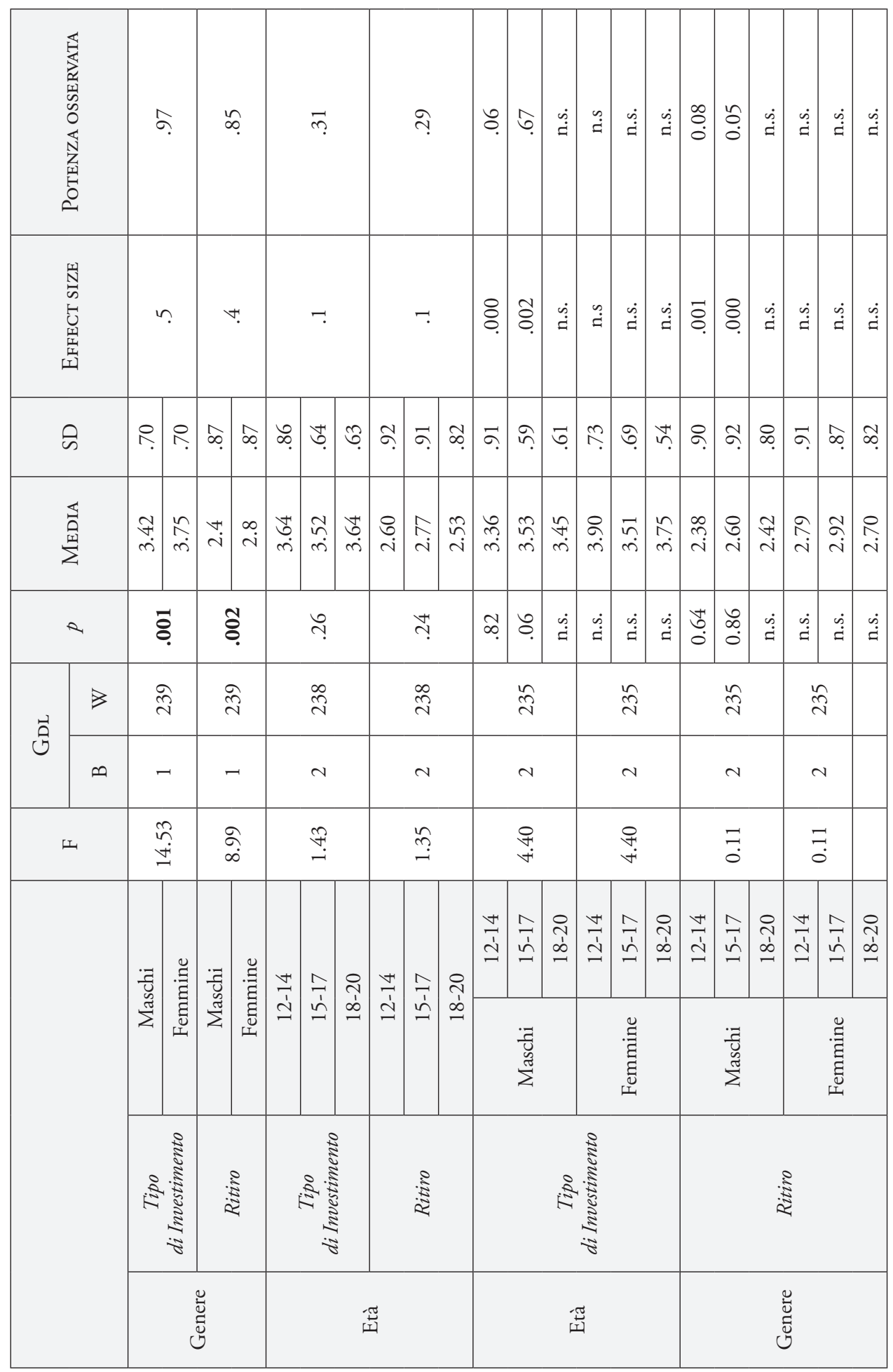




\section{ConClusioni}

Dalle analisi sono emersi risultati soddisfacenti per quanto riguarda l'attendibilità e la validità del questionario costruito e questo ci porta a ritenere che possa essere utilizzato, in futuro, come uno strumento agile e flessibile atto a misurare Modelli Culturali di utilizzo del servizio di counselling in diversi contesti.

Inoltre, le tre scale possono essere utilizzate sia singolarmente (come emerge nelle prime analisi fattoriali) al fine di esplorare specifiche rappresentazioni culturali del servizio di counselling orientate al Provocare, Affidarsi e Temere, sia connesse tra di loro (come emerge nella seconda analisi fattoriale) con lo scopo di esplorare specifiche dinamiche collusive tra studente e servizio di counselling. In questo secondo caso emergono, in particolare, due fattori.

Il Primo Fattore, denominato Tipo di Investimento, esprime un atteggiamento più attivo, caratterizzato principalmente da una propensione ad investire l'altro, il counsellor appunto, inteso come esperto, capace di contenere i propri problemi o i propri agiti, e per questo figura a cui affidarsi o da attaccare e provocare. E, dunque, implicita la simbolizzazione dell'altro come dotato di maggior potere, potere che può generare una forte dipendenza entro una relazione asimmetrica con un adulto percepito come competente. L'asimmetria e la competenza, se riconosciute ed accettate, sono in grado di favorire atteggiamenti di ricerca di aiuto, di affidamento, ma, se negate e non tollerate, possono dar vita ad atteggiamenti inibitori che ostacolano la ricerca di aiuto.

Questi dati sono in linea con altri studi presenti in letteratura che, con particolare riferimento all'investimento di natura provocatoria, hanno sottolineato come uno dei principali ostacoli alla ricerca di aiuto sia la preferenza che gli adolescenti mostrano nel gestire da soli o con i pari i problemi personali, per mostrarsi così più autonomi e indipendenti dagli adulti (Bacchini, Esposito, \& Affuso, 2009; Offer, Howard, Schonert, \& Ostrov, 1991; Kuhl, Jarkon-Horlick, \& Morrissey, 1997; Raviv et al., 2000 e 2009).

Il Secondo Fattore, denominato Ritiro, esprime, invece, un atteggiamento più passivo, di attesa, in cui l'ansia e la paura dell'altro rendono lo studente restio a rivolgersi al counsellor, inteso come una figura che spaventa, che giudica e, in ultima analisi, che valuta proprio come farebbe un insegnante. $\mathrm{E}$, dunque, implicita la simbolizzazione dell'altro come un adulto che intimorisce, di cui non ci si può fidare perché si teme che possa essere violata la propria riservatezza. Questo risultato è in accordo con quanto riferito in molti altri studi (Dubow, Lovko, \& Kausch, 1990; West et al., 1991; Wilson \& Deane, 2000; Wilson, Rickwood, Ciarrocchi, \& Deane, 2002; Sheffield, 
Fiorenza, \& Sofronoff, 2004), i quali sottolineano come un ostacolo alla ricerca di aiuto sia la mancanza di familiarità con la persona del counsellor. Da questo fattore, oltre all'imbarazzo e alla vergogna, emerge anche la dimensione della valutazione che sembra richiamare la dinamica relazionale attiva proprio all'interno dell'istituzione scolastica: infatti, sembra che la stanza di consultazione venga accomunata all'aula, il counsellor al docente, l'incontro ad un' interrogazione che spaventa, intimorisce, preoccupa e genera una paura dell'estraneo che non consente di valorizzare l'incontro con l'altro (Esposito, Freda, \& Franzese, 2010).

I risultati della MANOVA hanno posto in evidenza, in entrambi i fattori, una differenza tra ragazzi e ragazze. Questo dato è in accordo con altri studi rilevati in letteratura (Boldero \& Fallon, 1995; Schonert-Reichl \& Muller, 1996; Laitinen-Krispijn et al., 1999; Rickwood, 2005) secondo cui le ragazze sembrano avere un atteggiamento più positivo verso la ricerca di aiuto psicologico, una maggiore consapevolezza della necessità di aiuto e una maggiore fiducia negli operatori rispetto ai ragazzi (Leong \& Zachar, 1999). Inoltre, come sottolinea Maggiolini (2006) con riferimento al modo di manifestare i propri problemi: «Le ragazze tendono comunque a simbolizzarli, a parlarne, mentre i maschi sono meno portati ad ammettere di avere difficoltà personali, perché questo contrasta con un'immagine di ruolo sessuale che non tollera debolezze. Negano di avere difficoltà o le generalizzano come problema comune, e tendono ad agire per risolvere i problemi piuttosto di pensarci o parlarne con qualcuno».

In ragione proprio della possibilità che lo strumento offre di analizzare dinamiche affettive che connotano Modelli Culturali di uso del counselling, riteniamo che esso possa avere una serie di ricadute applicative nei diversi contesti scolastici.

Innanzitutto ISCOS, a differenza di altri questionari di valutazione del counselling o di analisi delle attitudini a rivolgersi al servizio, offre la possibilità di esplorare rappresentazioni culturali, intese come sistemi di significato intersoggettivamente costruiti, che mediano la stessa richiesta di partecipazione al servizio di counselling e che influiscono sulla sua efficacia in caso di frequentazione dello stesso. Come abbiamo avuto modo di sottolineare nell'introduzione del contributo, riteniamo che gli esiti del processo di counselling e la propensione ad utilizzarlo siano influenzati da tali dinamiche che vanno quindi analizzate al fine di progettare interventi mirati.

Inoltre, con specifico riferimento al terzo filone di studi sul counselling descritto, ISCOS consente di sopperire ad una carenza metodologica di strumenti quantitativi che permettano, da un lato, di esplorare le dinamiche collusive che connotano lo specifico oggetto counselling (al momento ancora non analizzato mediante una metodologia ISO), dall'altro di favorire rileva- 
zioni su più larga scala rese possibili dalla natura quantitativa dello strumento e dalla sua agilità di somministrazione (si pensi al numero ridotto di item e al riferimento allo stimolo grafico delle vignette presenti nel questionario).

ISCOS, inoltre, può essere somministrato, non solo a studenti che si sono già rivolti al counselling, ma anche a coloro che non ne hanno mai usufruito. Ciò può avvenire, ad esempio, durante una fase preliminare dell'intervento con obiettivi di conoscenza, sensibilizzazione e pubblicizzazione del servizio presso i vari contesti scolastici. Pertanto, esso può essere utilizzato come uno strumento di «diagnosi» del posizionamento isituzionale del servizio in grado di ri-orientare lo psicologo scolastico nel suo intervento e di promuovere uno spazio funzionale agli obiettivi degli studenti in ragione dello specifico contesto di appartenenza. L'utilizzo di ISCOS potrebbe consentire di istituire dei processi d'intervento informativo di «filtro» che permettano di aiutare lo studente a differenziare il ruolo e gli obiettivi del counsellor da quello del docente, promuovendo una rappresentazione del servizio quale spazio di sospensione del giudizio e della valutazione e, così facendo, di favorire negli studenti la propensione a cercare aiuto, aumentando la loro acceptance (Hwang, 1994; Wu, 1995) del servizio e di conseguenza la frequenza e l'utilizzo mirato.

Riteniamo, infatti, che l'ingente spesa pubblica effettuata nel promuovere servizi di counselling nel contesto scolastico nazionale e internazionale legittimi la necessità di ricorrere a strumenti che ne permettano un miglioramento in termini sia di aumento della frequentazione da parte degli studenti che ne sentono il bisogno (ma che spesso non vi si rivolgono a causa di dimensioni emozionali disfunzionali), sia di miglioramento della sua efficacia per quanti ne fanno un concreto utilizzo. Pensare e accogliere tali dimensioni emozionali potrà permettere al counsellor scolastico di comprendere in maniera più profonda e specifica quelli che sono gli obiettivi di sviluppo degli studenti di quello specifico contesto scolastico e rispondervi in maniera più consona e adeguata.

\section{LIMITI DELLO STRUMENTO E IMPLICAZIONI PER IL FUTURO}

Il questionario richiede ulteriori approfondimenti per verificarne l'affidabilità e la validità, ampliando e diversificando ulteriormente il campione per quanto riguarda la provenienza e l'indirizzo scolastico frequentato. Infatti, è stato finora somministrato in un'unica scuola; per cui i risultati ottenuti potrebbero essere stati influenzati dagli specifici Modelli Culturali operanti nella scuola in cui è stata effettuata la rilevazione. Inoltre, sarebbe anche 
interessante considerare altri metodi di verifica dell'attendibilità, come per esempio il metodo test-retest (Pedrabissi \& Santinello, 1997) al fine di determinare il grado di concordanza dei risultati ottenuti nelle due applicazioni, la stabilità dei punteggi e la non-casualità delle risposte.

Sebbene lo studio ci abbia portati ad eliminare la scala del Diffidare per la sua bassa affidabilità, sarebbe interessante, negli studi futuri, attraverso una differente procedura iniziale di selezione degli item narrativi, riproporre questo modello che, a differenza del Provocare, non colpisce tanto la regola del gioco (Carli \& Paniccia, 2011), quanto piuttosto la rappresentazione fantasmatica dell'altro come potenziale esperto in grado di fornire aiuto: ci si comporta, quindi, come se la fiducia nell'altro fosse venuta meno e come se ci si guardasse bene dall'esplorare relazioni nuove per timore di spiacevoli sorprese. Tale modello, dunque, ci potrebbe offrire una simbolizzazione diversa dell'altro rispetto ai modelli individuati, che potrebbe rivelarsi utile nella progettazione futura d'interventi all'interno dell'istituzione scolastica, fornendoci un quadro più completo e complesso del rapporto tra servizio di counselling e studente.

\section{RIFERIMENTI BIBLIOGRAFICI}

Ajzen, I. (2002). Constructing a TPB questionnaire: Conceptual and methodological considerations. http://www-unix.oit.umass.edu/-aizen/tpb.html.

Bacchini, D., Esposito, G., \& Affuso, G. (2009). Social experience and School Bullying. Journal of Community \& Applied Social Psychology, 19, 17-32. doi: 10.1002/casp.975.

Blos, P. (1962). L'adolescenza (trad. it). Milano: Franco Angeli, 1980.

Bolasco, S. (1999). Analisi multidimensionale dei dati. Metodi, strategie e criteri d'interpretazione. Roma: Carocci.

Boldero, J., \& Barry, F. (1995). Adolescent help-seeking: What do they get help for and from whom? Journal of Adolescence, 18(2), 193-209.

Bryan, J., Moore-Thomas, C., Day-Vines, N. L., Holcomb-McCoy, C., \& Mitchell, N. (2009). Characteristics of students who receive school counseling services: Implications for practice and research. Journal of School Counseling, $7(21), 1-29$.

Bury NHS Trust (2010). The Experience of Service Questionnaire (ESQ). Pennine Care NHS Foundation Trust. http://www.penninecare.nhs.uk/.

Busani, F. (2005). La consulenza psicologica a scuola. Dieci anni di ascolto a scuola. In A. Varani \& F. Forzani (a cura di), Star bene a scuola. Dieci anni di esperienze di educazione alla salute. Milano: Mediserve. 
Candela, A. (2005). Students' participation as co-authoring of school institutional practices. Culture \& Psychology, 11(3), 321-337.

Carli, R., Lancia, F., Paniccia, R. M., \& Pelagalli, M. F. (1997). Nuovi modelli di comunicazione e sviluppo territoriale. Psicologia Clinica, 3(2), 41-64.

Carli, R., \& Paniccia, R. M. (2002). L'Analisi Emozionale del Testo, uno strumento psicologico per leggere testi e discorsi. Milano: Franco Angeli.

Carli, R., \& Paniccia, R. M. (2003). Analisi della domanda. Teoria e tecnica dell'intervento in psicologia clinica. Bologna: Il Mulino.

Carli, R., \& Paniccia, R. M. (2011). La cultura dei servizi di salute mentale in Italia. Franco Angeli: Milano.

Carli, R., Paniccia, R. M., Di Ninni, A., Scala, V., Pagano, P., Giovagnoli, F., Bucci, F., Dolcetti, F., Bagnato, S., Sesto, C., Terenzi, V., \& Bonavita, V. (2009). La Cultura Locale dei Centri di Salute Mentale (CSM) in Italia. Rivista di Psicologia Clinica, 3, 1-60.

Carli, R., Paniccia, R. M., \& Salvatore, S. (2004). Limmagine dello psicologo in Toscana. Lo psicologo nella cultura della Regione Toscana. http://www.psicologia. toscana.it.

Carli, R., \& Salvatore, S. (2001). Limmagine della psicologia. Una ricerca sulla popolazione del Lazio. Roma: Edizioni Kappa.

Cavalieri, P. (2003). Misure. Metodologia integrata di misurazione della customer satisfaction, Atti del XXI Convegno Nazionale AICQ Qualità oggi: cosa cambia. Contributi per capire, Roma. http://www.spsonline.it.

Chireshe, R. (2011). School counsellors' and students' perceptions of the benefits of school guidance and counselling services in Zimbabwean secondary schools. Journal of Social Science, 29(2), 101-108.

Ciarrocchi, J., Wilson, C. J., Dean, F. P., \& Rickwood, D. (2003). Do difficulties with emotions inhibit help-seeking in adolescence? The role of age and emotional competence in predicting help-seeking intentions. Counselling Psychology Quarterly, 16(2), 103-120.

Cicognani, E. (2003). Psicologia sociale e ricerca qualitativa. Roma: Carocci.

Cooper, M., Rowland, N., McArthur, K., Pattison, S., Cromarty, K., \& Richards, K. (2010). Randomised controlled trial of school-based humanistic counseling for emotional distress in young people: Feasibility study and preliminary indications of efficacy. Child \& Adolescent Psychiatry \& Mental Health, 4(12), $1-12$.

Di Fabio, A. (2003). Counseling e relazione d'aiuto. Linee guida e strumenti per l'autoverifica. Firenze: Giunti.

Dolcetti, F., Giovagnoli, F., Paniccia, R. M., \& Carli, R. (2006). La cultura degli psicoterapisti e dei loro clienti a confronto, JADT 2006: 8es Journées internationales d'Analyse statistique des Données Textuelles. 
Dubow, E. F., Lovko, K. R., Jr., \& Kausch, D. F. (1990). Demographic differences in adolescents' health concerns and perceptions of helping agents. Journal of Clinical Child Psychology, 19, 44-54.

Egbochuku, E. O. (2008). Assessment of the quality of guidance and counseling services to students' adjustment in secondary schools in Edo state of Nigeria. Research Journal of International Studies, 8, 42-50.

Ercolani, A. P., Areni, A., \& Manetti, L. (1998). La ricerca in psicologia. Roma: Carocci.

Esposito, G., Freda, M. F., \& Franzese, C. (2010). Profili di utilizzo del counselling individuale a scuola: tra ansie di valutazione paralizzanti e dinamiche attive di affidamento. Psichiatria dell'Infanzia e dell'Adolescenza, 77(3), 695-714.

Esposito, G., Zaccaro, A., \& Freda, M. F. (2009). Il Centro di Informazione e Consulenza (CIC): uno spazio di cerniera tra relazione educativa e relazione di aiuto. Psicologia Scolastica, 8(1), 111-131.

Eyo, M. B., Joshua, A. M., \& Esuong, A. E. M. (2010). Attitude of secondary school students towards guidance and counseling services in cross river state. Edo Journal of Counselling, 3(1), 87-99.

Fairchild, T. N., \& Seeley, T. J. (1996). Evaluation of school psychological services: A case illustration. Psychology in the Schools, 33, 46-55.

Fischer, E. H., \& Farina, A. (1995). Attitudes toward seeking professional psychological help: A shortened form and considerations for research. Journal of College Student Development, 36(4), 368-373.

Freda, M. F., Esposito, G., \& La Montagna, F. (2009). Profili di utilizzo del counselling a scuola: tra sfogo, crisi ed opportunità di crescita. Psichiatria dell'Infanzia e dell'Adolescenza, 75(3/4), 569-581.

Gattini, D. M. (2005). L'adolescente allo sportello d'ascolto. In A. Varani \& F. Forzani (a cura di), Star bene a scuola. Dieci anni di esperienze di educazione alla salute. Milano: Mediserve.

Giovagnoli, F., Dolcetti, F. R., \& Paniccia, R. M. (2008). Le attese e le valutazioni sulla psicoterapia dal punto di vista degli psicoterapeuti e dei clienti. Rivista di Psicologia Clinica, 3, 334-353.

Giovagnoli, F., Giuliano, S., \& Paniccia, R. M. (2009). Il vissuto sulla psicoterapia in un gruppo di persone che non ne hanno mai fatto esperienza. Rivista di Psicologia Clinica, 1, 50-67.

Goh, M., Xie, B., Herting Wahl, K., Zhong, G., Lian, F., \& Romano, J. L. (2007). Chinese students' attitudes toward seeking professional psychological help. International Journal for the Advancement of Counselling, 29, 187-202.

Hwang, C. (1996). An investigation of the understanding level and attitudes for helpseeking from college students. Unpublished master's Thesis, National Chung Hua Normal University, Chung Hua city, citato in Lin, 2002. 
Kuhl, J., Jarkon-Horlick, L., \& Morrissey, R. F. (1997). Measuring barriers to helpseeking behavior in adolescents. Journal of Youth and Adolescence, 26(6), 637650 .

Iannaccone, A., Komatsu, K., \& Marsico, P. (Eds.). (2002). Crossing boundaries. Intercontextual dynamics between family and school. Charlotte, NC: Information Age Publication.

Laitinen-Krispijn, S., Van der Ende, J., Wierdsma, A. I., \& Verhulst, F. C. (1999). Predicting adolescent mental health service use in a prospective record-linkage study. American Academy of Child and Adolescent Psychiatry, 38, 1073-1081.

Leong, F. T. L., \& Zachar, P. (1999). Gender and opinions about mental illness as predictors of attitudes toward seeking professional psychological help. British Journal of Guidance and Counselling, 27, 123-132.

Lin, Y. (2002). Taiwanese university students' conceptions of counseling. Journal of Contemporary Psychotherapy, 31(3), 199-211.

Linell, P. (2009). Rethinking language, mind and world dialogically: Interactional and contextual theories of sense making. Charlotte, NC: Information Age Publishing.

Lonborg, S. D., \& Bowen, N. (2004). Counsellors, communities and spirituality: Ethical and multicultural considerations. Professional School Counselling, 7(5), 318-331.

Losito, G. (2004). L'analisi del contenuto nella ricerca sociale. Milano: Franco Angeli.

Lunenburg, F. C. (2010). School guidance and counselling services. Schooling, 1(1), $1-9$.

Madera, P. (2007). I Centri d'Ascolto. Risultati di una ricerca svolta nelle scuole medie del veronese orientale. Psicologia e Scuola, 136, 55-64.

Maggiolini, A. (2006). Counseling a scuola. Milano: Franco Angeli.

McKenzie, K., Murray, G. C., Prior, S., \& Stark, L. (2010). An evaluation of a school counselling service with direct link to Child and Adolescent Mental Health (CAMH) services. British Journal of Guidance \& Counselling, 39(1), 67-82.

Ngale, I. F. (2009). Students' perception of school counseling government high schools in Bamenda sub-division, Cameroon. http://www.thefreelibrary.com.

Offer, D., Howard, K. L., Schonert, K. A., \& Ostrov, E. (1991). To whom do adolescents turn for help? Differences between disturbed and non disturbed adolescent. American Academy of Child and Adolescent Psychiatry, 30, 623-630.

Ozabaci, N. (2011). Turkish students' metaphorical conceptualizations of school counsellors. South African Journal of Psychology, 41(1), 29-37.

Pedrabissi, L., \& Santinello, M. (1997). I test psicologici. Bologna: Il Mulino.

Prior, S. (2011). Overcoming stigma: How young people position themselves as counselling service users. Sociology of Health \& Illness, 20(10), 1-17. 
Raviv, A., Sills, R., \& Wilansky, P. (2000). Adolescents' help-seeking behaviour: The difference between self- and other-referral. Journal of Adolescence, 23, 721740.

Raviv, A., Vago-Gefen, I., \& Schachter Fink, A. (2009). The personal service gap: Factors affecting adolescents' willingness to seek help. Journal of Adolescence, 32(3), 483-499.

Rickwood, D. J., Deane, F. P., \& Wilson, C. J. (2007). When and how do young people seek professional help for mental health problems? The Medical Journal of Australia, 187(7), 35-39.

Salvatore, S. (2012). Social life of the sign: Sensemaking in society. In J. Valsiner (Ed.), The Oxford handbook of culture and psychology. Oxford: Oxford University Press.

Salvatore, S., \& Scotto di Carlo, M. (2005). Lintervento psicologico per la scuola. Modelli, metodi, strumenti. Roma: Carlo Amore.

Salvatore, S., Valsiner, J., Travers, J., \& Gennaro, A. (Eds.). (2010). Yearbook of idiographic science, Vol. 3. Roma: Firera Publishing Group.

Sayal, K. (2006). Annotation: Pathways to care for children with mental health problems. Journal of Child Psychological Psychiatry, 47, 649-659.

Schonert-Reichl, K. A., \& Muller, J. R. (1996). Correlates of help-seeking in adolescence. Journal of Youth and Adolescence, 25, 705-731.

Sheffield, J. K., Fiorenza, E., \& Sofronoff, K. (2004). Adolescents' willingness to seek psychological help: Promoting and preventing factors. Journal of Youth and Adolescence, 33(6), 495-507.

Takamura, S., Oshima, I., Yoshida, K., \& Motonaga, T. (2008). Factors related to attitudes toward seeling professional psychological help among Japanese junior high and high school students. Yonago Acta Medica, 51, 39-47.

Twigg, E., Barkham, M., Bewick, B. M., Mulhern, B., Connell, J., \& Cooper, M. (2009). The young person's CORE: Development of a brief outcome measure for young people. Counseling and Psychotherapy Research, 9, 160-168.

Valsiner, J., \& Rosa, A. (Eds.). (2007). The Cambridge handbook of sociocultural psychology. Cambridge: Cambridge University Press.

Venuleo, C., Salvatore, S., Mossi, P., Grassi, R., \& Ruggeri, R. (2008). The didactic relationship in the changing world. Outlines for a theory of the reframing setting. European Journal of School Psychology, 5(2), 151-180.

Vogel, D. L., Wade, N. G., \& Hackler, A. H. (2007). Perceived public stigma and the willingness to seek counseling: The mediating roles of self-stigma and attitudes towards counseling. Journal of Counseling Psychology, 54(1), 40-50.

West, J. S., Kayser, L., Overton, P., \& Saltmarsh, R. (1991). Student perceptions that inhibit the initiation of counseling. School Counselor, 39, 77-83. 
Whiston, S. C., Lee Tai, W., Rahardja, D., \& Eder, K. (2011). School counselling outcome: A meta-analytic examination of interventions. Journal of Counselling and Development, 89, 37-55.

Wilson, C. J., \& Deane, F. P. (2000). Exploring help-negation: Is it a manifestation of help-seeking barriers? Manuscript submitted for publication. In Wilson, Deane, Ciarrocchi, \& Rickwood, 2005.

Wilson, C. J., Deane, F. P., Ciarrocchi, J., \& Rickwood, D. (2005). Measuring helpseeking intentions: Properties of the General Help-Seeking Questionnaire. Canadian Journal of Counselling / Revue Canadienne de Counseling, 39(1), 15-28.

Wilson, C. J., Rickwood, D., Ciarrochi, J., \& Deane, F. P. (2002). Adolescent barriers to seeking professional psychological help for personal-emotional and suicidal problems, Conference Proceedings of the $9^{\text {th }}$ Annual Conference for Suicide Prevention, June 2002, Sydney.

Winograd, G., \& Shick Tryon, G. (2009). Counselling expectations among students in an opportunity program: Dispositional and cultural influences. Journal of Counselling and Development, 87(4), 438-449.

Wu, J. (1995). The effects of gender and problem type on university students' attitudes. Unpublished master's Thesis, National Chung Hua Normal University, Chung Hua city, citato in Lin, 2002. 


\section{APPENDICE}

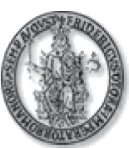

UNIVERSITÀ DEGLI STUDI DI NAPOLI «FEDERICO II»

DIPARTIMENTO DI SCIENZE RELAZIONALI "G. IACONO»

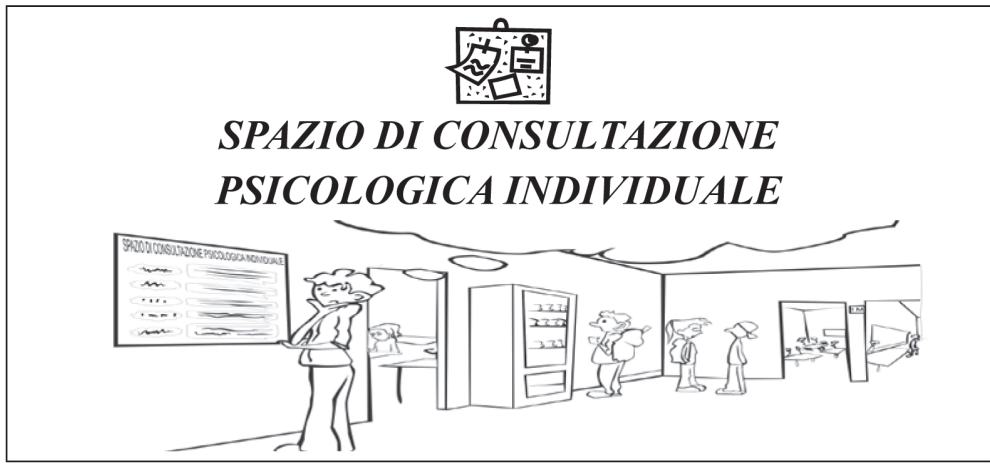

Il questionario che ti presentiamo si inserisce all'interno di una ricerca condotta dal Dipartimento di Psicologia dell'Università di Napoli «Federico II», il cui obiettivo è esplorare la rappresentazione che i giovani studenti hanno dello spazio di consultazione psicologica individuale attivo nelle scuole. Tu sei stato scelto per partecipare a questa ricerca.

Le tue risposte ci permetteranno di comprendere l'utilità che tale spazio ha per voi studenti. Non ci sono risposte "giuste» o «sbagliate», ma solo risposte più o meno vicine a ciò che si pensa o si sente.

La regola più importante che ti chiediamo di tener presente è : «La prima risposta è quella che conta».

Il questionario è rigorosamente anonimo.

Ti presenteremo delle vignette, sarai invitato a metterti nei panni del protagonista e a raccontarci i tuoi pensieri.

Spesso ti verrà chiesto di fare una scelta tra diverse alternative e può capitare che non ti riconosca completamente in nessuna di esse. In questo caso ti chiediamo di «forzare» la tua posizione e dare comunque una risposta.

\section{Grazie per la collaborazione}

Codice Questionario $\llcorner\perp \perp$ 


\section{IN PRIMO LUOGO, TI FAREMO ALCUNI ESEMPI, PER CAPIRE INSIEME COME RISPONDERE AL QUESTIONARIO}

Esempio: Il protagonista della vignetta sta correndo verso la porta per segnare il goal della vittoria. Prova a metterti nei suoi panni: «Sei lì, che corri verso la meta, ma un avversario si frappone tra te e il tuo obiettivo, marcandoti stretto, quali potrebbero essere i tuoi pensieri, in questo specifico momento?». Di seguito sono riportati alcuni pensieri possibili, poni una «X» sul numero che meglio corrisponde al tuo grado di accordo o disaccordo, tenendo sempre ben presente che:

\section{1 = Per nulla d'accordo \\ 2 = Poco d'accordo \\ 3 = Né d'accordo né in disaccordo \\ 4 = Molto d'accordo \\ 5 = $\underline{\text { Moltissimo d'accordo }}$}

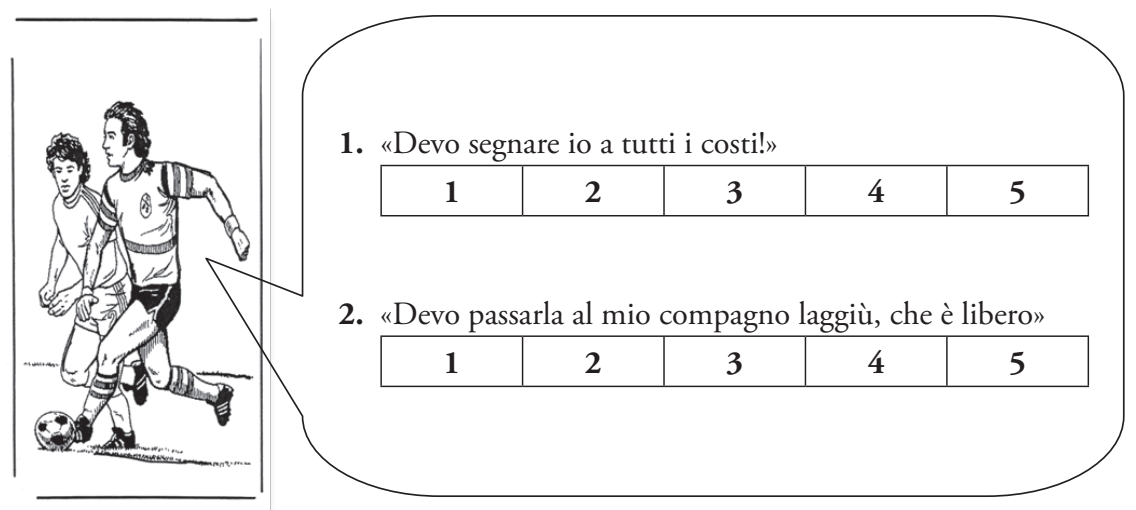


Esempio: La protagonista della vignetta è appena tornata da una giornata di shopping folle, in cui ha speso molti soldi. Prova a metterti nei suoi panni: "Sei lì, con tutte quei pacchi in mano e stai per tornare a casa, dove dovrai spiegare ai tuoi genitori perché hai speso così tanti soldi, quali potrebbero essere i tuoi pensieri, in questo specifico momento?». Di seguito sono riportati alcuni pensieri possibili, poni una «X» sul numero che meglio corrisponde al tuo grado di accordo tenendo sempre ben presente che:

\section{$1=\underline{\text { Per nulla d'accordo }}$}

2 = Poco d'accordo

$3=\underline{\text { Né d'accordo né in disaccordo }}$

$4=\underline{\text { Molto d'accordo }}$

5 = Moltissimo d'accordo

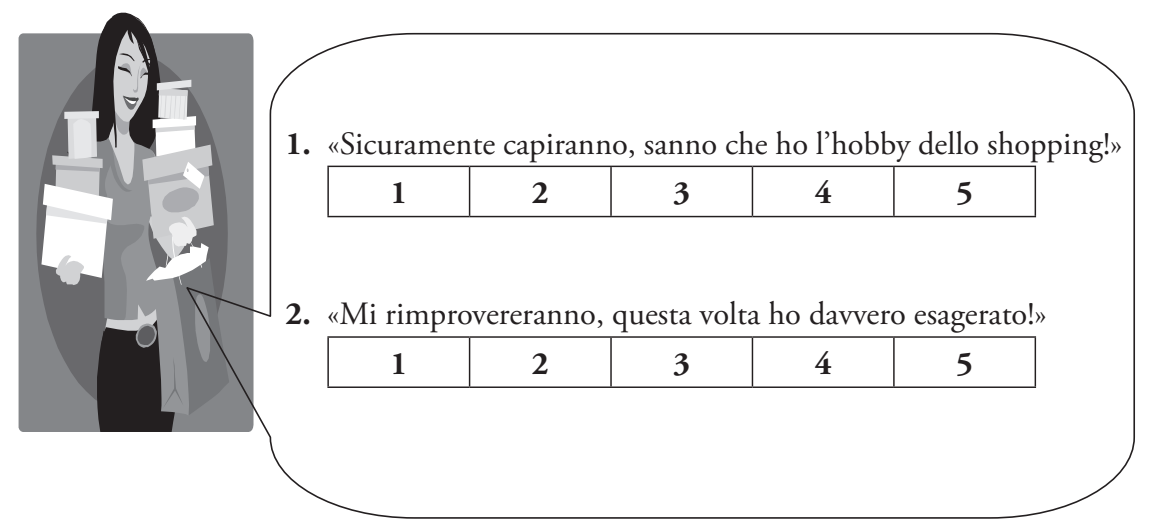




\section{ORA ENTRIAMO NEL VIVO DEL QUESTIONARIO, BUON LAVORO!}

Situazione I: L'ENTRATA. Il protagonista della vignetta sta entrando nella stanza dello spazio di consultazione psicologica individuale. Prova a metterti nei suoi panni: «Sei lì, davanti alla porta e la stai per aprire, quali potrebbero essere i tuoi pensieri in quello specifico momento?». Di seguito sono riportati alcuni pensieri possibili, indica con una "X» il tuo grado di accordo o disaccordo con ciascuno di essi, tenendo sempre ben presente che:

1 = Per nulla d'accordo; 2 = Poco d'accordo; 3 = Né d'accordo né in disaccordo; 4 = Molto d'accordo; = Moltissimo d'accordo

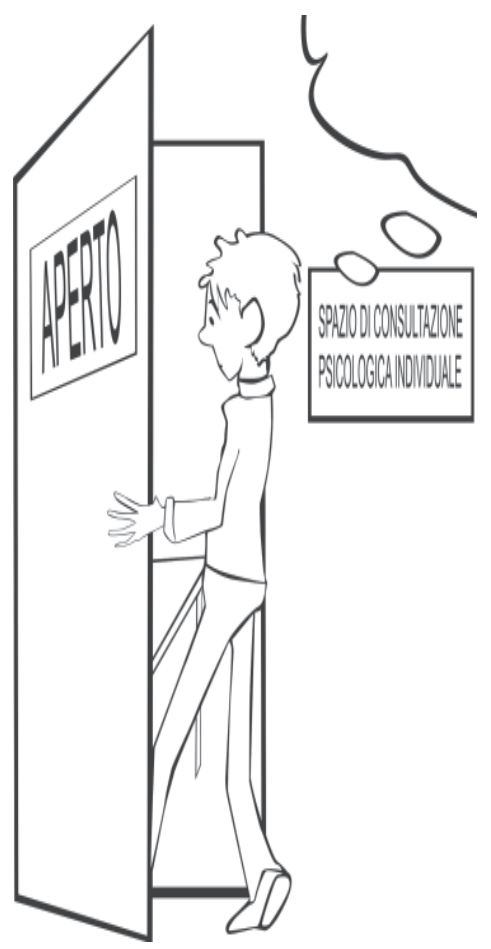

1. "Che imbarazzo!! Chissà cosa penserà di me!»

\begin{tabular}{|l|l|l|l|l|}
\hline 1 & 2 & 3 & 4 & 5 \\
\hline
\end{tabular}

2. «Ecco, è il momento giusto per parlare dei miei problemi e sfogarmi senza essere giudicato"

\begin{tabular}{|l|l|l|l|l|}
\hline 1 & 2 & 3 & 4 & 5 \\
\hline
\end{tabular}

3. "Voglio proprio vedere cosa mi dice!»

\begin{tabular}{|l|l|l|l|l|}
\hline 1 & 2 & 3 & 4 & 5 \\
\hline
\end{tabular}

4. «Entro, ma tanto non ho nessun problema!»

\begin{tabular}{|l|l|l|l|l|}
\hline 1 & 2 & 3 & 4 & 5 \\
\hline
\end{tabular}

5. «Mah! Chissà se riusciranno ad aiutarmi»

\begin{tabular}{|l|l|l|l|l|}
\hline 1 & 2 & 3 & 4 & 5 \\
\hline
\end{tabular}

6. "Che vergogna! Speriamo che nessuno mi veda entrare»

\begin{tabular}{|l|l|l|l|l|}
\hline 1 & 2 & 3 & 4 & 5 \\
\hline
\end{tabular}

7. «Finalmente qualcuno che mi può aiutare a capire meglio i miei pensieri»

\begin{tabular}{|l|l|l|l|l|}
\hline 1 & 2 & 3 & 4 & 5 \\
\hline
\end{tabular}

8. «Entriamo va, così perdo l'ora di lezione!»

\begin{tabular}{|l|l|l|l|l|}
\hline 1 & 2 & 3 & 4 & 5 \\
\hline
\end{tabular}


Situazione II: L'INCONTRO. La protagonista della vignetta è entrata nella stanza dello spazio di consultazione psicologica individuale. Eccola, seduta di fronte alla psicologa. Prova a metterti nei suoi panni : «Sei lì, seduta sulla sedia, di fronte alla psicologa, quali potrebbero essere i tuoi pensieri, in quello specifico momento?». Di seguito sono riportati alcuni pensieri possibili, indica con una "X»" il tuo grado di accordo o disaccordo con ciascuno di essi, tenendo sempre ben presente che:

\section{1 = Per nulla d'accordo; 2 = Poco d'accordo; 3 = Né d'accordo né in di- saccordo; 4 = $\underline{\text { Molto d'accordo; }}$ = $\underline{\text { Moltissimo d'accordo }}$}

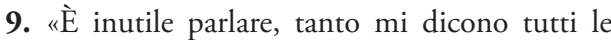
stesse cosen

\begin{tabular}{|l|l|l|l|l|}
\hline 1 & 2 & 3 & 4 & 5 \\
\hline
\end{tabular}

10. «Chissà se posso fidarmi!»

\begin{tabular}{|l|l|l|l|l|}
\hline 1 & 2 & 3 & 4 & 5 \\
\hline
\end{tabular}

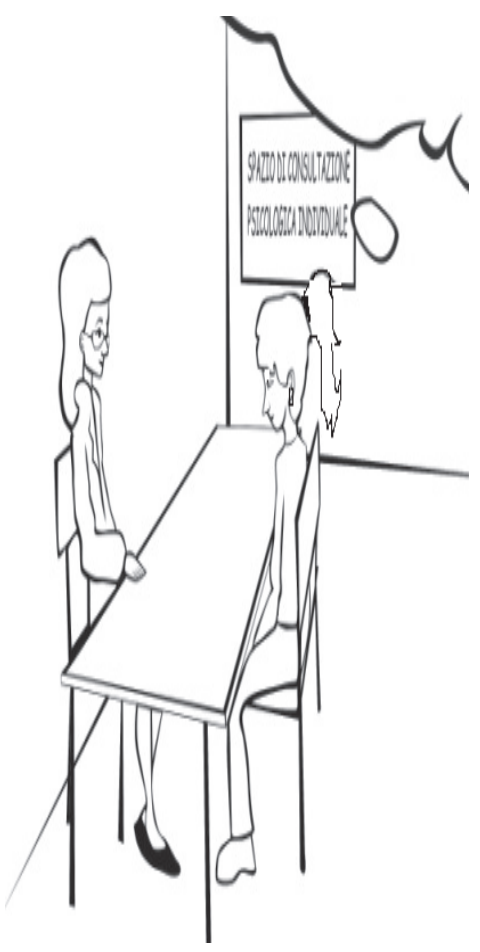

11. «Buongiorno! Ho un problema, non so cosa fare e vorrei un consiglio"

\begin{tabular}{|l|l|l|l|l|}
\hline 1 & 2 & 3 & 4 & 5 \\
\hline
\end{tabular}

12. “Mi vergogno un po' di esprimere le mie emozioni e raccontare di me: speriamo di non fare brutte figure»

\begin{tabular}{|l|l|l|l|l|}
\hline 1 & 2 & 3 & 4 & 5 \\
\hline
\end{tabular}

13. "Che ansia, speriamo che mi faccia qualche domanda perché io non so proprio da dove incominciare»

\begin{tabular}{|l|l|l|l|l|}
\hline 1 & 2 & 3 & 4 & 5 \\
\hline
\end{tabular}

14. "A lei non dico la verità, perché dei miei problemi ne parlo con la mia famiglia o con i miei amici, mi capiscono sicuramente di più»

\begin{tabular}{|l|l|l|l|l|}
\hline 1 & 2 & 3 & 4 & 5 \\
\hline
\end{tabular}

15. "Non so se posso aprirmi, in fondo è una persona che non conosco!»

\begin{tabular}{|l|l|l|l|l|}
\hline 1 & 2 & 3 & 4 & 5 \\
\hline
\end{tabular}

16. «Mi fa piacere parlare con una persona che ha più esperienza di me»

\begin{tabular}{|l|l|l|l|l|}
\hline 1 & 2 & 3 & 4 & 5 \\
\hline
\end{tabular}


Situazione III: L'USCITA. Il protagonista della vignetta ha terminato il colloquio e sta per uscire dalla stanza dello spazio di consultazione psicologica individuale. Prova a metterti nei suoi panni: «Sei lì, stai uscendo dalla stanza, dopo aver parlato con la psicologa, quali potrebbero essere i tuoi pensieri, in quello specifico momento?». Di seguito sono riportati alcuni pensieri possibili, indica con una « $\mathbf{X}$ » il tuo grado di accordo o disaccordo con ciascuno di essi, tenendo sempre ben presente che:

1 = Per nulla d'accordo; 2 = Poco d'accordo; 3 = Né d'accordo né in disaccordo; 4 = Molto d'accordo; = Moltissimo d'accordo

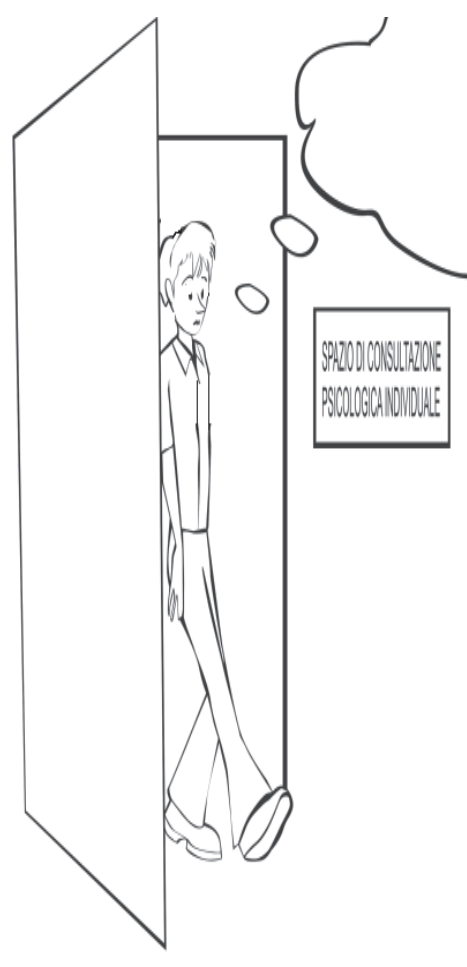

17. «Lo sapevo non è servito a niente. Sto peggio di prima»

\begin{tabular}{|l|l|l|l|l|}
\hline 1 & 2 & 3 & 4 & 5 \\
\hline
\end{tabular}

18. "Mi sento più rilassato e con le idee più chiare»

\begin{tabular}{|l|l|l|l|l|}
\hline 1 & 2 & 3 & 4 & 5 \\
\hline
\end{tabular}

19. «Non so se la prossima volta ritornerò»

\begin{tabular}{|l|l|l|l|l|}
\hline 1 & 2 & 3 & 4 & 5 \\
\hline
\end{tabular}

20. «Non ce l'ho fatta proprio a parlare anche se volevo sfogarmi. Avevo troppa vergogna»

\begin{tabular}{|l|l|l|l|l|}
\hline 1 & 2 & 3 & 4 & 5 \\
\hline
\end{tabular}

21. «Mi sento scettico, chissà per quale motivo fanno queste sedute»

\begin{tabular}{|l|l|l|l|l|}
\hline 1 & 2 & 3 & 4 & 5 \\
\hline
\end{tabular}

22. «Ah! Meno male che è finita! Mi stavo proprio annoiando!»

\begin{tabular}{|l|l|l|l|l|}
\hline 1 & 2 & 3 & 4 & 5 \\
\hline
\end{tabular}

23. «Mi sento sollevato ora che ho parlato dei miei problemi invece di tenermeli dentro»

\begin{tabular}{|l|l|l|l|l|}
\hline 1 & 2 & 3 & 4 & 5 \\
\hline
\end{tabular}

24. "Che imbarazzo, non sapevo proprio che dire!»

\begin{tabular}{|l|l|l|l|l|}
\hline 1 & 2 & 3 & 4 & 5 \\
\hline
\end{tabular}




\section{TI CHIEDIAMO, IN CONCLUSIONE, ALCUNI TUOI DATI}

25. Sesso:

$\square$ Maschio

$\square$ Femmina

26. La tua età:

27. Classe:

28. Indica, per favore, il luogo dove vivi:

29. Professione della madre

(se pensionata o deceduta specificare l'ultimo lavoro)

30. Professione del padre

(se pensionato o deceduto specificare l'ultimo lavoro)

31. Titolo di studio della madre

32. Titolo di studio del padre

33. Sei mai stato allo spazio di consultazione psicologica individuale, presente nella tua scuola?
$\square$ Sì
$\square$ No

GRAZIE PER LA COLLABORAZIONE 


\section{RiassunTO}

Il lavoro si propone di costruire e validare un nuovo questionario, denominato ISCOS (Indicatori di Sviluppo del COunselling Scolastico), volto a misurare specifici profili di utilizzo del counselling a scuola. Tali profili, definiti del Provocare, Diffidare, Temere e Affidarsi al servizio, cosi come emersi in ricerche esplorative precedenti (Freda et al., 2009; Esposito et al., 2010), sono stati concettualizzati quali modelli simbolici e culturali (Carli \& Paniccia, 2003 e 2011) di rappresentazione del servizio. Si è cercato altresi di indagare le possibili associazioni tra tali modelli di utilizzo e l'influenza di variabili come l'età e il genere degli studenti. ISCOS è stato somministrato a 241 studenti $(F=115 ; M=126)$ di età compresa tra i 13 e i 20 anni $(M=16.23$; ds = 1.81), frequentanti una Scuola Media di secondo grado della città di Napoli in cui è attivo da diversi anni il servizio di counselling. L'analisi fattoriale esplorativa ha evidenziato la stabilità di due fattori (Tipo di Investimento e Ritiro) che spiegano il 37\% della varianza totale. Infine, per entrambi i fattori si è riscontrata una significativa differenza solo per quanto riguarda la variabile genere. Il questionario ha mostrato buone qualità psicometriche. Esso si presenta come uno strumento innovativo volto ad analizzare specifiche dimensioni emozionali sottese ai modelli di uso individuati. Tuttavia, si ritengono necessarie ulteriori indagini diversificando e ampliando ulteriormente il campione per quanto riguarda la provenienza e l'indirizzo scolastico.

Parole chiave: Counselling Scolastico, Modelli di uso, Prevenzione, Profili di utilizzo, Questionario, Validazione. 